\title{
At your own peril: An ERP study of voluntary task set selection processes in the medial frontal cortex
}

\author{
BIRTE U. FoRSTMANN AND K. RICHARD RIDDERINKHOF \\ University of Amsterdam, Amsterdam, The Netherlands \\ AND \\ Jochen KaISER AND Christoph BLedowsKi \\ Johann Wolfgang Goethe University, Frankfurt am Main, Germany
}

\begin{abstract}
A tool that is commonly used to investigate selection among different alternatives in a changing environment is the task-switching paradigm. Functional neuroimaging has pointed out a role for the posterior medial frontal cortex and the posterior parietal cortex in the voluntary selection of task sets. In the present study, we set out to investigate the temporal dynamics of these agency-related processes (in task choice vs. no-choice conditions) using event-related brain potentials (ERPs). The results revealed agency-related modulations of a series of ERP components, including (1) an early parieto-occipital activation, taken to reflect the evaluation of choice versus no choice; (2) a subsequent medial frontal expression of the voluntary selection between task sets; (3) a CNV-like sustained negativity in preparation for the target; (4) a target-induced N210-P210 complex, taken to reflect early sensory-perceptual processing; and (5) a target-induced $\mathrm{P} 3$, associated with the evaluation of the stimulus and its designated response vis-à-vis the chosen versus competing task sets. Together, these results indicate that the opportunity to choose between tasks invokes activity originating from the medial frontal cortex, associated with voluntary task set selection, but also activation at different time points in a number of other brain areas, not necessarily captured by functional neuroimaging.
\end{abstract}

A considerable literature has begun to document the neural bases of action selection, as well as task switching. Little is understood, however, about the neural bases of agency (the capacity to make choices and to enact those choices in the world) in the voluntary selection of actions and task sets. Initial evidence has begun to point to the role of the medial frontal cortex (MFC) in voluntary action selection (electrophysiological studies) and task set selection (functional neuroimaging studies). In the present eventrelated brain potential (ERP) study, our aim was to investigate electrocortical expressions of the role of the MFC in voluntary task set selection. In particular, we examined the ERP components elicited by an agency stimulus that informs the subject about which tasks he or she can choose from. In situations of choice (as compared with no choice), we expected to observe modulations of the stimulusinduced medial frontal ERP sites, reflecting voluntary task set selection. More exploratively, we expected this frontal modulation to be preceded by modulations of ERP components that reflect perceptual encoding and evaluation of the agency stimulus and to be followed by modulations of ERP components that reflect preparation for the upcoming target. The ERPs elicited by a subsequent target stimulus itself might also be modulated by agency.

\section{Voluntary Selection}

The endeavor of modern neurosciences is to find neural mechanisms that underlie the control of human cognition (Carter et al., 1998; Duncan \& Owen, 2000). Cognitive control refers to the ability to orchestrate, direct, and adaptively modulate cognitive processes in accordance with internal goals and intentions and with (sometimes rapidly changing) external demands. There is broad evidence for the involvement of the prefrontal cortex (PFC) in cognitive control and, therefore, in our ability to flexibly adapt our behavior (Miller \& Cohen, 2001; Ridderinkhof, van den Wildenberg, Segalowitz, \& Carter, 2004).

Cognitive control can be characterized in terms of a dimension that has been referred to as proactive versus reactive, endogenous versus exogenous, top down versus bottom up, cue induced versus target induced, and the like (cf. Braver, Gray, \& Burgess, 2007). Proactive control enforces internally driven plans and goals of action (e.g., when we recall the steps of how to prepare our favorite dish), whereas reactive control allows actions to be guided by salient external events (e.g., a red traffic light makes us hit the brakes).

The task-switching paradigm has been used as a powerful tool to investigate both proactive and reactive control

B. U. Forstmann, b.u.forstmann@uva.nl 
components (see, e.g., Koch, 2003; Meiran, 1996; Rogers \& Monsell, 1995). In the task-switching paradigm, subjects have to alternate between simple discrimination tasks (e.g., color or shape). Often, in neuroimaging studies, an external cue is used to signal the relevant task to be performed. These studies have shown that the lateral PFC seems to play a crucial role for the updating of task set representations (Brass, Derrfuss, Forstmann, \& von Cramon, 2005; Brass \& von Cramon, 2004; Derrfuss, Brass, Neumann, \& von Cramon, 2005). However, what happens if subjects are not directly instructed which task to perform but, instead, can select the task themselves? This question refers to agency and the neural bases of voluntary task set selection.

There is growing evidence from neuroimaging studies that voluntary selection processes are subserved by an area in the posterior part of the MFC - most prominently, in the rostral cingulate zone (RCZ; see, e.g., Picard \& Strick, 1996; Ullsperger \& von Cramon, 2001). Using functional magnetic resonance imaging (fMRI), the RCZ was shown to be involved also in the voluntary selection of task sets (Forstmann, Brass, Koch, \& von Cramon, 2006). In this study, agency was manipulated by varying the number of tasks from which the subjects could choose between a forced condition (no choice) and two voluntary selection conditions (two or three choices). Whereas the RCZ was sensitive to agency (exhibiting stronger activation in the choice vs. the no-choice conditions), it did not reveal selection-specific activation differences between three and two choices.

The same pattern was also found in the posterior parietal cortex - in particular, in the posterior part of the intraparietal sulcus (pIPS) and the superior parietal lobe (SPL). In general, activation in the IPS and the SPL has been reported by a number of neuroimaging studies in which task-related control has been investigated with the task-switching paradigm (Brass \& von Cramon, 2002; Forstmann, Brass, Koch, \& von Cramon, 2005; Ruge et al., 2005; Wylie, Javitt, \& Foxe, 2004). Furthermore, they have been consistently activated in various tasks involving spatially directed attention (Corbetta, Kincade, Ollinger, McAvoy, \& Shulman, 2000; Corbetta \& Shulman, 2002; Fink, Dolan, Halligan, Marshall, \& Frith, 1997). A common feature among the visuospatial tasks in the experiments revealing comparable posterior parietal activations is that subjects were asked to direct their attention covertly to peripheral target locations in order to detect or discriminate a stimulus (Fink et al., 1997; Kastner, Pinsk, De Weerd, Desimone, \& Ungerleider, 1999; Nobre et al., 1997).

In general, there is growing evidence from the electrophysiological literature for a fronto-parietal network to be involved in the internal or voluntary selection of task sets (Rushworth, Passingham, \& Nobre, 2002, 2005). However, in view of the constraints imposed by the poor temporal resolution of fMRI, the nature and dynamics of the processes implemented in frontal and parietal regions remain to be uncovered. For instance, it remains open whether activation in frontal areas might precede and lead activation in parietal areas when a task set is voluntarily selected. There is growing evidence that prefrontal areas send a biasing signal to parietal regions to implement concrete stimulus-response (S-R) mappings (Miller \& Cohen, 2001; Tomita, Ohbayashi, Nakahara, Hasegawa, \& Miyashita, 1999).

\section{The Present Study}

In the present study, we recorded electroencephalographic (EEG) signals while subjects performed a modified task-switching paradigm. Agency was varied between a forced condition (no choice) and two voluntary choice conditions (choosing between two and three tasks). The subjects always had to switch between task sets. That is, the task that was chosen on the present trial was never a valid option for choice on the next trial. To achieve this constraint, four simple discrimination tasks were introduced. A trial always started with a cue indicating which task(s) could potentially be chosen. After a task was selected, a target stimulus was presented that the subjects responded to according to their preceding task selection. Cue and target were separated by a long and constant cue-target interval (CTI) of 2,000 msec. Finally, a probe stimulus appeared, and the subjects now indicated with a second response which task they had actually selected and responded to with their first response.

The aim of the analysis of ERPs derived from the EEG was to differentiate between cue- and target-related neural activity in order to delineate the role of proactive and reactive processes involved in agency. We expected to find proactive agency-related modulations of ERP components linked to the presentation of the cue. More specifically, in situations of choice (as compared with no choice), we expected the voluntary selection of task sets to be reflected in ERP modulations at medial frontal electrode sites. Preceding this index of voluntary selection, the perceptual encoding and evaluation of choice versus no-choice cues might yield more posterior ERP modulations. Subsequent to the medial frontal modulation, differential preparation for the upcoming target in choice versus no-choice conditions might be expressed in the contingent negative variation (CNV). The ERPs elicited by a subsequent target stimulus itself may also be modulated by agency.

In ERP studies of task switching, even though a task cue helps the subject prepare for the upcoming task, the ERP components elicited by the target stimulus still differ between conditions. For instance, as compared with task repetitions, task alternations tend to be associated with smaller P3 components (e.g., Kieffaber \& Hetrick, 2005). Likewise, in the present study, even though the agency cue allowed the subject to choose an upcoming task and prepare for it, the ERP components elicited by the target stimulus might still differ between conditions (choice vs. no choice).

\section{METHOD}

\section{Subjects}

Nineteen healthy volunteers were recruited. We obtained written consent from all 19 subjects prior to the EEG measurement. All the subjects had normal or corrected-to-normal vision. No subject had a history of neurological, major medical, or psychiatric disorder. The data of 5 subjects were excluded from the analysis due to strong eye movements and technical problems. The remaining 14 subjects were 
9 females and 5 males (age: $M=25.07$ years, $S D=6.96$ ), who were all right-handed (self-report).

\section{Behavioral Task}

Four cues, each associated with one of four simple discrimination tasks - that is, color, orientation, size, and line tasks - were introduced. The cues were foveally presented in a $2 \times 2$ grid at the beginning of each trial with a visual angle of $2.8^{\circ}$ (Figure 1). Each quadrant of the grid contained a semantic abbreviation for one of the four tasks - that is, "FAR" for Farbe, which corresponded to color; "SPI" for Spitze, which corresponded to orientation; "GRÖ" for Größe, which corresponded to size; and "LIN" for Linie, which corresponded to line. The locations of the cues were balanced across subjects. There were 16 possible cue location mappings, which were randomly assigned to the subjects so that each mapping was presented only once. In order to instruct the subjects as to which task set could be chosen, the quadrants were either bold, indicating that this task could be chosen, or not, indicating that this task was not available for choice. After a constant interval of 2,000 msec, the target was presented. This target was multivalent; that is, the target contained one value for each discrimination task. There were 16 possible values of a target. In accord with their choice of task, the subjects had to respond to the target with either the index or the middle finger of the right hand. In order to check for accuracy and to give valid feedback, the four semantic abbreviations of the four tasks were presented again. This time, they were horizontally aligned while the order of the semantic cues was pseudorandomized. The subjects now indicated, with their left hand, using a key that was spatially compatible with one of the horizontally aligned semantic abbreviations, which task they had actually chosen and responded to with their first response. In the following, we will refer to the horizontally aligned cues as the probe stimuli. The randomization of the probe stimuli ensured that the subjects did not prepare for the second response during the decision time. Finally, feedback for wrong responses, misses, or correct responses was presented for $500 \mathrm{msec}$.

The design consisted of one independent variable, the degrees of freedom $(d f \mathrm{~s})$, which varied from 1 to 3 . The trials started with a fixation cross that was presented for $50 \mathrm{msec}$, followed by the $2 \times 2$ grid, which was always presented for $2,000 \mathrm{msec}$. Thus, the CTI was held constant. Then the target was presented until a response was made with the right hand or until the response interval exceeded $2,500 \mathrm{msec}$. After the first response had been given, the probe stimuli were presented. These remained on the screen until the second response was made with the left hand or until the response interval exceeded 3,000 msec. One trial lasted $10 \mathrm{sec}$.

The experiment consisted of two blocks with 270 experimental trials each, resulting in approximately 19 trials of the combination of the task and the $d f_{\mathrm{s}}(1-3)$. There were 14 different combinations of tasks and $d f(d f 1,4$ combinations; $d f 2,6$ combinations; $d f 3,4$

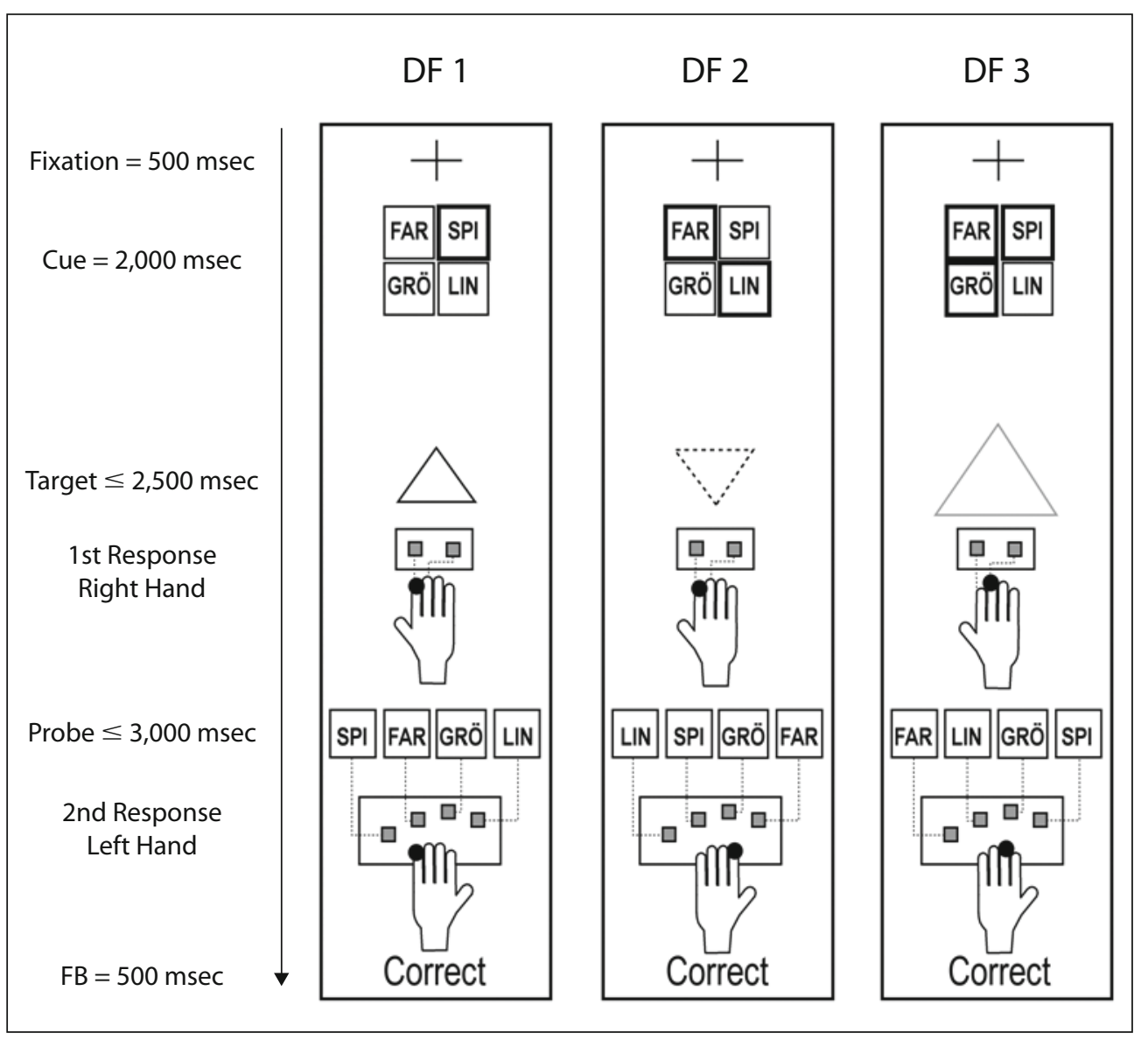

Figure 1. Paradigm: Schematical drawing of the different conditions. Degrees of freedom were varied between the forced condition ( $d f 1$ ) and the choice conditions ( $d f 2$ and $d f 3$ ). These were indicated by bold lines. Instructions for tasks were given by German abbreviations in the $2 \times 2$ grid with "FAR" (color), "SPI" (orientation), "GRÖ" (size), and "LIN" (line). The target and probe stimuli were presented until a response was given or an interval of 2,500 or 3,000 msec, respectively, was exceeded. FB, feedback. 
combinations). Even though we could not introduce prerandomized task sequences, due to the manipulation of free choice, we defined subsets of each combination of tasks and the $d f$. Trials were pseudorandomly chosen from each subset. This ensured that each combination was presented with approximately equal frequency. Moreover, on each trial, the subjects had to select between new task sets and could not select the same task set again - that is, no repetition trials. Furthermore, it is important to note that the subsequent trial of the df 3 condition was not constrained to the one task that was not valid for choice. This was achieved by an adaptive procedure implemented in the program. The experiment lasted about $60 \mathrm{~min}$.

\section{EEG Acquisition and Data Analysis}

EEG measurements were conducted in an electrically shielded and sound-attenuated chamber. Stimuli were displayed using Presentation Software (www.neurobs.com) and a TFT monitor with a resolution of $1,024 \times 768$ and a refresh rate of $60 \mathrm{~Hz}$. The distance between the computer screen and the subjects was about $100 \mathrm{~cm}$. The stimulation monitor was placed outside the cabin behind an electrically shielded window. EEG activity was recorded using a 128-channel QuickAmp amplifier (www.brainproducts.com) and custom-made electrode caps with 124 electrodes, mounted in an elastic cap with equal spacing over the whole head and symmetric placements between both hemispheres. The inferior row of electrodes was placed $20 \%$ below the international $10-20$ system, thus providing sufficient coverage of the lower parts of the head. Two additional electrodes were placed on the infraorbital ridges of the left and right eyes, respectively, and two further electrodes were placed on the neck below the edge of the electrode cap.

All channels were recorded with an average reference, a forehead ground electrode and impedances of less than $15 \mathrm{k} \Omega$. No filter was used during recordings. The EEG was digitized with a sampling rate of $500 \mathrm{~Hz}$. A spatial digitizer was used to record the individual spherical coordinates of each electrode and of three fiducial landmarks (the left and right preauricular points and the nasion) to allow for a correct coregistration of scalp topographies over subjects.

EEG data were analyzed with the BESA software package (www besa.de). Prior to signal averaging, individual EEG data were corrected for eye blinks, using an adaptive correction method (Ille, Berg, \& Scherg, 2002) and were filtered with a band-pass of $0.1-15 \mathrm{~Hz}$ (zero phase).

In order to describe the general temporospatial pattern of the neuronal processes associated with the voluntary selection of task sets, we first analyzed continuous EEG epochs of 3,500-msec duration including a 500-msec precue baseline, a 2,000-msec CTI, and a $1,000-\mathrm{msec}$ posttarget interval. To investigate the differential effects due to the experimental conditions and to obtain a better signal-tonoise ratio, we focused on EEG epochs closely related to cue and target events, respectively.

Thresholds were used to discard artifactual epochs with a signal level exceeding $75 \mu \mathrm{V}$ from the segment baseline in any channel or a slew rate exceeding $75 \mu \mathrm{V} / \mathrm{msec}$. Data from 5 subjects were discarded because of pronounced eye movement artifacts, which resulted in fewer than 30 remaining epochs out of 90 possible trials per experimental condition. On average, $88.6 \%$ of all epochs in 14 subjects were retained after artifact rejection. The retained EEG epochs on correct trials were averaged to cue and to target onset separately for each of the three $d f$ conditions $(d f 1, d f 2$, and $d f 3)$.

The ERPs to cue events were calculated in a 1,000-msec time epoch including a 200-msec precue baseline. Target-related ERPs were analyzed in a 1,500-msec time epoch, and a baseline correction for the period $500 \mathrm{msec}$ before target onset was performed (further labeled as target-related ERPs with pretarget baseline). Whereas this analysis focused on the time interval that followed the target stimulus presentation, we performed an additional target-related ERP analysis that addressed the activity differences in the time interval prior to target onset (i.e., during preparation for the target). Here, we used $200 \mathrm{msec}$ before cue onset for the baseline correction in the target-related ERP (further labeled as target-related ERPS with precue baseline).
For analogous comparisons with the previous fMRI study using the same task (Forstmann et al., 2006), a combined $d f 2$ and $d f 3$ condition was computed by averaging the ERP responses from the $d f 2$ and $d f 3$ conditions. This procedure was further justified by comparable behavioral effects in these two conditions (see the Behavioral Performance section)

After averaging, the individual ERP data (128 electrodes) in the $d f 1$ and the combined $d f 2$ and $d f 3$ conditions were interpolated to a standardized 101-electrode configuration using spherical spline interpolation. This allowed us to account for interindividual differences in head shape and electrode placements when performing group-level statistical tests.

To compare the ERP activity between the $d f 1$ and the combined $d f 2$ and $d f 3$ conditions, individual difference ERP waves at nine representative electrodes (three midline electrodes [Fz, Cz, and Pz] and six lateral electrodes [left, F7, C5, and P7; right, F8, C6, and P8]) were calculated by subtracting the ERP of the combined $d f 2$ and $d f 3$ condition from that of the $d f 1$ condition. To test for significant differences between the two choice conditions, $95 \%$ confidence intervals for these difference ERP waves were determined using the bootstrap bias-corrected and adjusted (BCa) method (Efron \& Tibshirani, 1993). The difference between the $d f 1$ and the combined $d f 2$ and $d f 3$ conditions was considered significant if the confidence interval of the difference ERP wave did not include zero for a minimum of 25 continuous time points $(50 \mathrm{msec})$.

\section{RESULTS}

\section{Behavioral Performance}

For the analyses of error rates and reaction times (RTs), two-tailed Student's $t$ tests for the relevant comparisons were used. Error rates and RTs are displayed in Figure 2. The overall accuracy was $97.13 \%$. The error rates revealed no significant effects $(t \mathrm{~s}<1)$ between conditions.

The RTs for the target response revealed a significant effect for the $d f 1$ condition $(615 \mathrm{msec})$ versus the $d f 2$ condition $(719 \mathrm{msec})[t(13)=2.5, p<.05]$ and versus the $d f 3$ condition $(730 \mathrm{msec})[t(13)=2.8, p<.05]$, indicating higher RTs for the conditions in which the subjects could voluntarily choose a task (combined $d f 2$ and $d f 3=$ $725 \mathrm{msec}$ ), as compared with the no-choice condition $(615 \mathrm{msec})$. There was no significant difference between choice conditions [ $d f 3$ vs. $d f 2 ; t(13)=1.13, p>.20]$. Since the subjects were instructed that the response to the probe stimuli was unspeeded and only relevant for giving valid feedback, the probe RTs were not analyzed.

\section{Selection Strategies}

We tried to equalize the frequency of task types in combination with the $d f$ s. Therefore, it is important to note that the interpretation of specific selection strategies is highly constrained (see also the Method section). Furthermore, the subjects always had to switch between task sets so that the task set that was chosen on the present trial was never valid for choice on the next trial.

However, to test for the potential occurrence of specific selection strategies, several post hoc analyses were performed. The dependent measure was the number of trials that were chosen for each particular task. The independent variables were (1) the task type to account for a preference in task selection and (2) the cue location to account for a preference in location selection. Both analyses revealed no significant effect $(t \mathrm{~s}<1)$. 

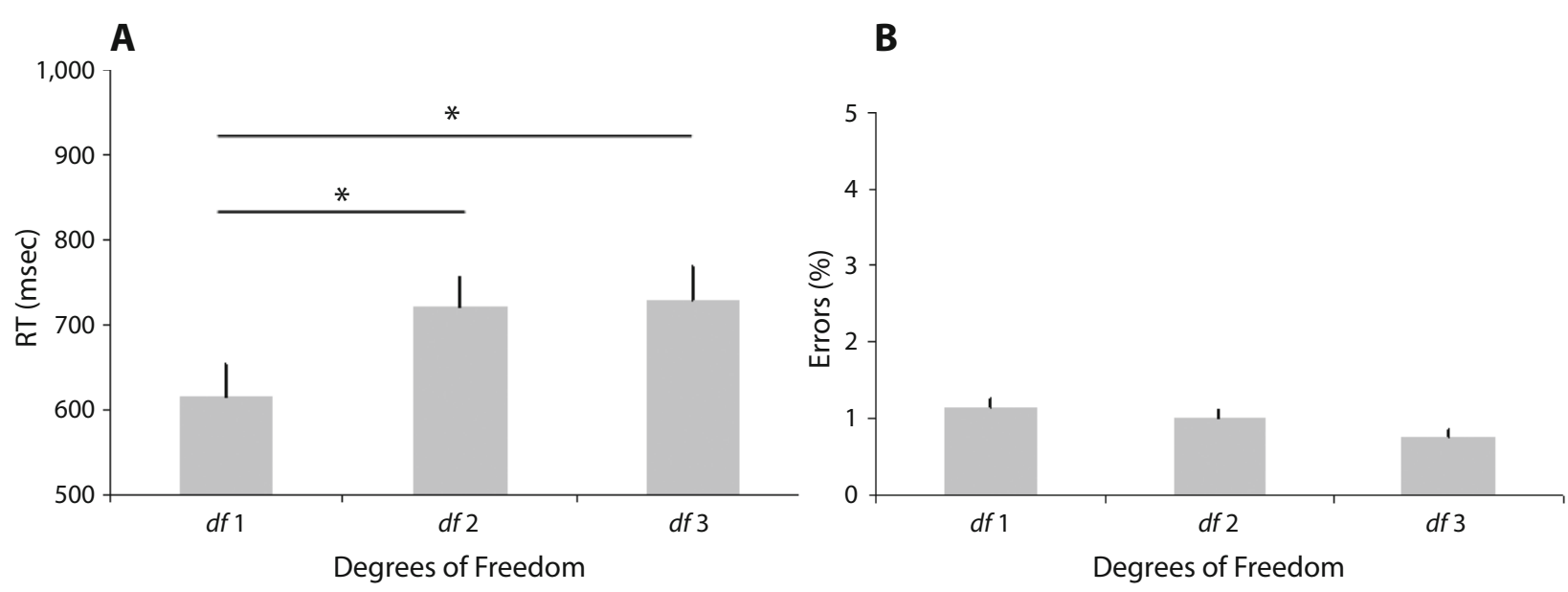

Figure 2. Behavioral data. (A) Mean reaction times (RTs) for the target response. (B) Percentages of errors as a function of degrees of freedom $(d f 1, d f 2$, and $d f 3)$, averaged for the $d f 2$ and $d f 3$ conditions.

\section{Congruency of S-R Mappings}

Comparable to the analysis conducted for the RTs acquired during the fMRI experiment (see also Forstmann et al., 2006), we were interested whether incongruent S-R mappings in the $d f 2$ and $d f 3$ conditions might have elicited the present data pattern. More specifically, in choice conditions, it is possible that the stimulus dimensions that are mapped onto different responses (incongruent trials) elicit higher response conflict, as compared with stimulus dimensions that are mapped onto the same response (congruent trials). The results of a nonparametric Wilcoxon test for two paired samples revealed no main effect for congruency or the $d f \mathrm{~s}(t \mathrm{~s}<1)$. This was also the case for the interaction between congruency and the $d f \mathrm{~s}$. In sum, the results indicate that congruency of valid S-R mappings is unlikely to explain the present data pattern.

\section{ERP Results}

The ERP time courses (see Figure 3) in the analyzed epoch including the 500-msec precue baseline, 2,000 -msec $\mathrm{CTI}$, and 1,000-msec posttarget interval, superimposed for all electrode sites, summarize the main ERP responses as- sociated with the different task phases. Several ERP components could be distinguished on the basis of their peak latencies and scalp topographies. The processing of the cue stimulus was associated with an early bilateral positivity over occipital electrode sites, peaking about $110 \mathrm{msec}$ after cue presentation, corresponding to the visual P1 ERP component. This occipital positivity was followed by a brief positive deflection with a maximum at electrode Pz. Furthermore, the cue stimulus elicited a pronounced positive P2-like ERP response with a maximum at frontocentral sites. This was followed by a broadly distributed P3-like ERP response at centro-parietal electrode sites.

During the CTI, a sustained activity was observed, with a negative deflection maximal at centro-parietal sites and a positivity at fronto-lateral sites, starting about $1,500 \mathrm{msec}$ after cue onset and reaching its maximum about $0-100 \mathrm{msec}$ after target onset.

The presentation of the target stimulus elicited a negative deflection after about $200 \mathrm{msec}$ at bilateral parietooccipital electrodes corresponding to the N1 ERP component. As with the cue related interval, a subsequent, broadly distributed ERP response was observed mainly

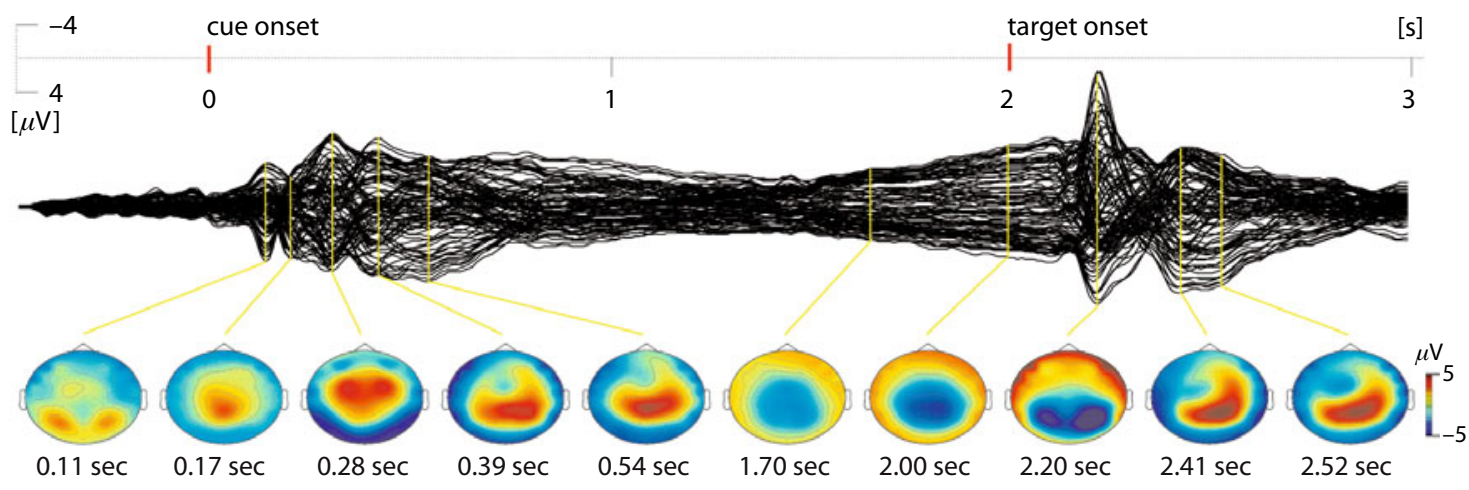

Figure 3. Superimposed grand average ERP waveforms (at 101 standard electrode sites interpolated from 128 recorded electrodes) to cue (at 0 sec) and target stimulus (at $2 \mathrm{sec}$ ) for the $d f 1$ condition and the corresponding spline-interpolated topographical maps of scalp voltage calculated at the latencies indicated by the yellow lines. 
at centro-parietal electrode sites corresponding to the P3 complex. Note that the more fronto-central part of this ERP component appeared lateralized to the right hemisphere.

Cue-related ERPs and target-related ERP (precue baseline). Selected electrode recordings and scalp topographies in Figures 4-6 show the different ERP responses for the two experimental conditions (combined $d f 2$ and $d f 3$, and $d f 1$ ). Table 1 lists the exact time intervals for the significant differences between the combined $d f 2$ and $d f 3$ versus $d f 1$ conditions at nine selected electrodes as revealed by the bootstrap statistics (for details, see the Method section). In addition, the difference maps (bottom rows of the right-hand panels in Figures 4-6) highlight the scalp distribution of the difference ERP responses (combined $d f 3$ and $d f 2$ minus $d f 1$ ) at corresponding time intervals of significant differences between the conditions in question. In particular, in the cue-related interval, we observed an increased transient ERP deflection for the $d f 1$

Table 1

Timing Intervals Showing Significant Differences in the ERP Signal During the Cue and the Target Phases

\begin{tabular}{ccc}
\hline & $\begin{array}{c}\text { Time Intervals With } \\
\text { Significant Differences }\end{array}$ \\
\cline { 2 - 3 } & Onset & Offset \\
\hline
\end{tabular}

\begin{tabular}{lcl}
\multicolumn{3}{c}{ Cue-Related ERP } \\
F7 & n.s. \\
Fz & n.s. \\
F8 & n.s. \\
C5 & n.s. & \\
Cz & 538 & 693 \\
C6 & n.s. & \\
P7 & n.s. & \\
Pz & 628 & 718 \\
P8 & 339 & 397
\end{tabular}

Target-Related ERP (Precue Baseline)

$\begin{array}{rrr}\text { F7 } & -194 & -39 \\ \text { Fz } & \text { n.s. } & \\ \text { F8 } & \text { n.s. } & \\ \text { C5 } & \text { n.s. } & \\ \text { Cz } & \text { n.s. } & \\ \text { C6 } & \text { n.s. } & \\ \text { P7 } & \text { n.s. } & -284 \\ \text { Pz } & -349 & -188 \\ & -260 & 149\end{array}$

Target-Related ERP (Pretarget Baseline)

$\begin{array}{lll}\text { F7 } & 268 & 586 \\ & 639 & 752\end{array}$

$\begin{array}{lll}\mathrm{Fz} & 84 & 252\end{array}$

$\begin{array}{lll}\text { F8 } & \text { n.s. } & \\ \text { C5 } & 217 & 637\end{array}$

$\begin{array}{ll}673 & 654\end{array}$

$\mathrm{Cz} \quad 194 \quad 674$

C6 $145 \quad 674$

P7 $174 \quad 242$

$\mathrm{Pz} \quad 332 \quad 450$

$478 \quad 673$

$859 \quad 1,000$

P8

n.s.

Note-Shown are onsets and offsets (in milliseconds) of the time intervals with significant differences between the combined $d f 2$ and $d f 3$ and the $d f 1$ conditions, as determined by the bootstrap BCa method (see the Method section). condition at about $350-400 \mathrm{msec}$ over the right parietooccipital electrode (P8) and at about 550-700 msec over the middle central electrode sites $(\mathrm{Cz})$ (see Figure 4).

In the target-related interval with the precue baseline (see Figure 5), the statistical analysis focused on the time interval around target stimulus processing (500 $\mathrm{msec}$ prior to 200-msec posttarget stimulus onset). The $d f 1$ condition showed a stronger negative deflection, which was most pronounced over middle parietal electrode sites in a time window between $350 \mathrm{msec}$ before and $150 \mathrm{msec}$ after target stimulus onset, probably reflecting preparation for target stimulus processing.

Target-related ERP (pretarget baseline). In the time interval after target stimulus presentation (target-related ERP with pretarget baseline; see Figure 6), we observed significant differences in several subsequent ERP deflections at frontal (F7 and Fz), central (C5, Cz, and C6) and parietal (P7 and $\mathrm{Pz}$ ) electrode sites. The earliest effect, at about 180-230 msec posttarget onset, was observed over frontal and left parietal sites (Fz and PO7), which was followed by a late centro-parietal effect (about 600-1,000 msec) with stronger responses for the $d f 1$ condition.

\section{DISCUSSION}

In the present study, we investigated the temporal dynamics of voluntary selection processes using ERPs. In the following, we will discuss the differential electrocortical expressions of proactive and reactive neural activity in choice conditions, as compared with no-choice conditions, as elicited by the cue, in anticipation of the target, and after presentation of the target.

\section{Cue-Related and Anticipatory-Related Activation}

The presentation of the cue revealed (among others) a broad ERP response over parietal and central-frontal electrode sites starting about $350 \mathrm{msec}$ after cue presentation and lasting about $400 \mathrm{msec}$, presumably representing the P3 complex. It has been proposed that the P3 is not a unitary phenomenon but the result of the activity of generators that are widely distributed in space and time (Kok, 2001; Verleger, 1997). Recent source localization studies combining ERP and fMRI data support this idea, indicating several frontal, parietal, and temporal P3 ERP generators (Bledowski et al., 2006; Bledowski et al., 2004; for a review, see Nieuwenhuis, Aston-Jones, \& Cohen, 2005). Interestingly, during the P3 time window, we found differential activities (choice vs. no-choice conditions) at parieto-occipital electrodes at about 350-400 msec and, somewhat later (at about $550-700 \mathrm{msec}$ ), also at medial frontal sites $(\mathrm{Cz})$. Moreover, prior to the target presentation, we observed a sustained negative deflection over centro-parietal sites, with higher amplitudes for the nochoice condition.

In general, the finding for these activation sites appears to resemble the findings from a previous fMRI study in which activation was found in the posterior parietal cortex, as well as in the RCZ (Forstmann et al., 2006). This fronto-parietal network was interpreted as reflecting the 

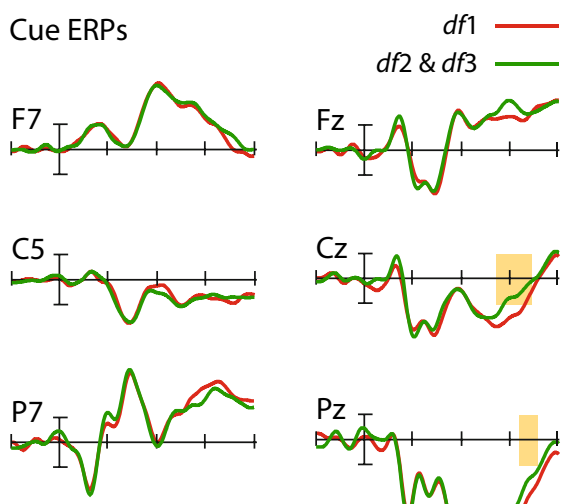
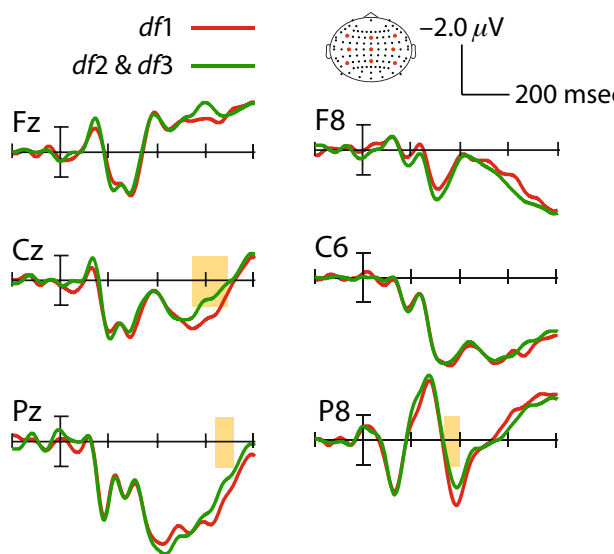

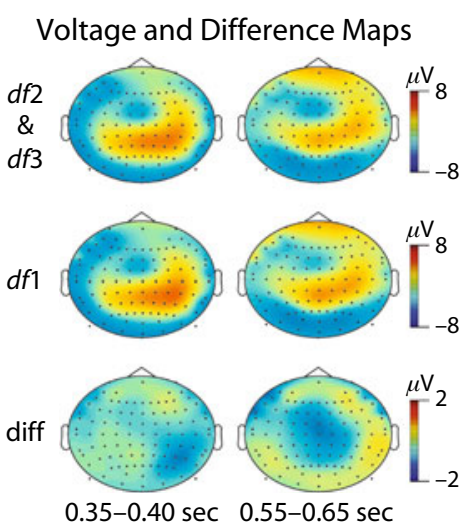

Figure 4. Separate cue-related ERP waveforms. Separate waveforms for $d f 1$ (red) and combined $d f 2$ and $d f 3$ (green) at nine selected electrodes (left panel). Yellow bars indicate time windows with significant differences between the combined $d f \mathbf{2}$ and $d f 3$ and the $d f 1$ conditions as determined by the bootstrap BCa method (see the Method section). Right panel: Spline-interpolated voltage topographical maps for the combined $d f \mathbf{f}$ and $d f 3$ and the $d f 1$ conditions and difference maps [diff ( $d f \mathbf{2}$ and $d f \mathbf{3}$ minus $d f$ 1)] at representative time points of significant differences between conditions in question.

voluntary selection of task sets in an all-or-none mannerthat is, independently of the number of choices. Although the present ERP effects resemble the activation sites of the previously conducted fMRI experiment, they show a reversed pattern with more activation for the no-choice condition than for the choice conditions.

It is a common finding in the ERP and fMRI literature that a more complex and demanding task may produce lower ERP amplitudes (e.g., P300; see Kok, 2001), whereas the opposite pattern is observed in the BOLD fMRI signal. Such opposite effects of BOLD and ERP amplitudes have also been observed in combined fMRI and EEG studies (e.g., Bledowski et al., 2006; Foucher, Otzenberger, \& Gounot, 2003; Huettel et al., 2004). The exact nature of these opposite ERP/fMRI effects remains unresolved. Several explanations have been proposed, such as an open versus a closed spatial layout of the ERP source, canceled versus intact phase-resetting contributions to the ERP, or different effects of the activity of inhibitory interneurons on EEG and fMRI activity (for a detailed discussion, see Bledowski et al., 2006). Another possibility is that smaller ERP responses could have resulted from a larger variability ( jitter) of ERP deflections across individual trials in the choice than in the no-choice conditions. It is possible that in the combined $d f 2$ and df 3 condition, the subjects mentally considered several alternatives. From trial to trial, the free selection of one of these alternatives could have taken place at different time points after cue onset, thus increasing the variability of the ERPs reflecting the selection process. This increased in-

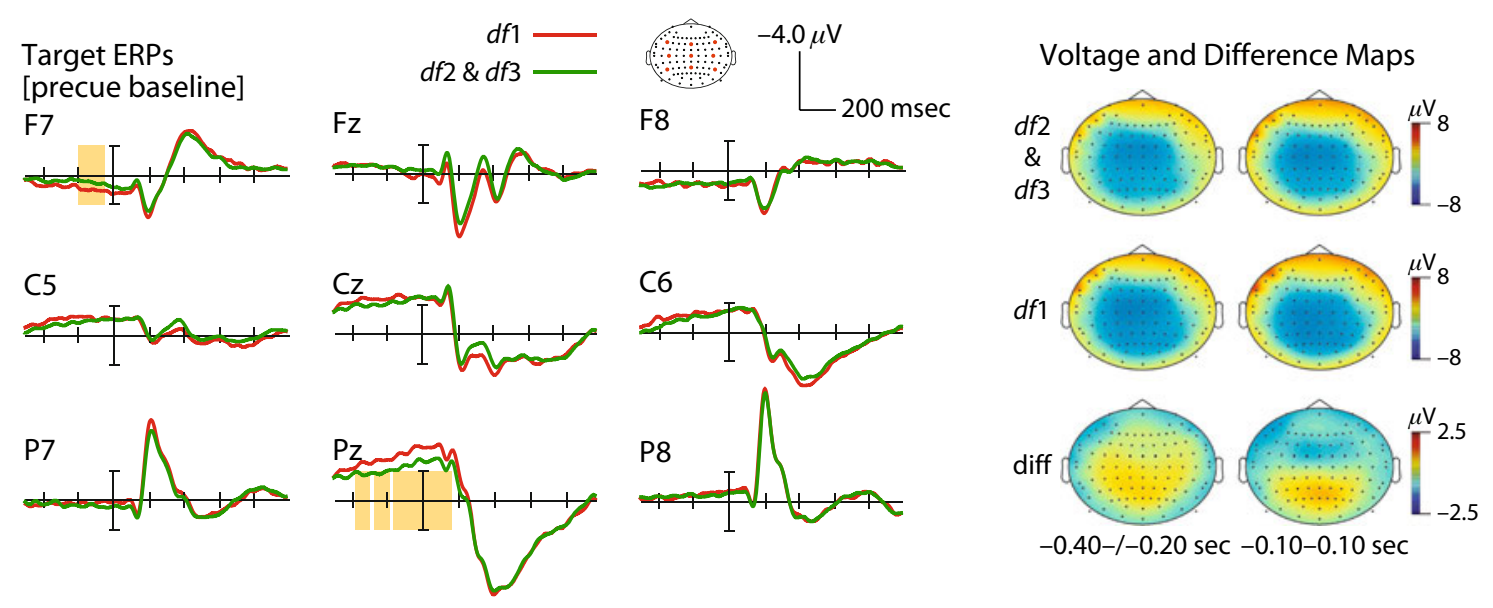

Figure 5. Separate target-related ERP waveforms (precue baseline). Separate waveforms for $d f 1$ (red) and combined df 2 and $d f 3$ (green) at nine selected electrodes (left panel). Yellow bars indicate time windows with significant differences between the combined $d f \mathbf{2}$ and $d f 3$ and the $d f 1$ conditions as determined by the bootstrap BCa method (see the Method section). Right panel: Spline-interpolated voltage topographical maps for the combined $d f \mathbf{2}$ and $d f 3$ and the $d f 1$ conditions and difference maps [diff ( $d f \mathbf{2}$ and $d f 3$ minus $d f 1)$ ] at representative time points of significant differences between conditions in question. 


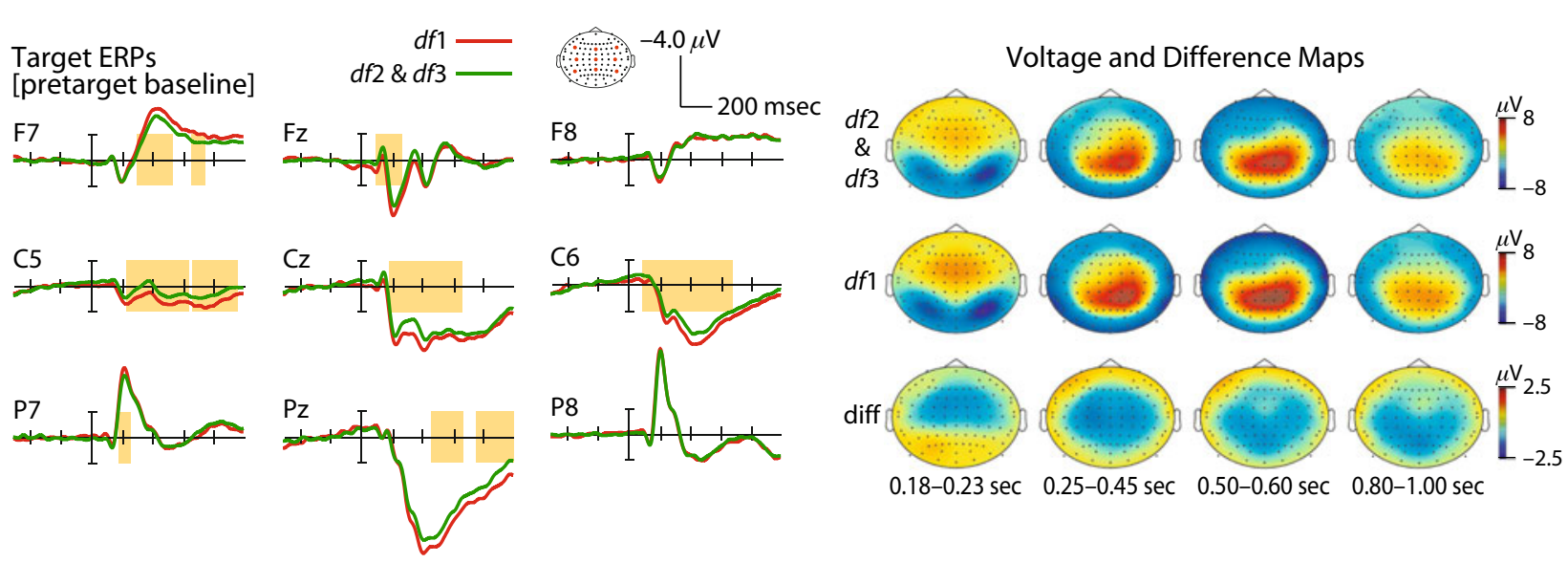

Figure 6. Separate target-related ERP waveforms (pretarget baseline). Separate waveforms for $d f \mathbf{f}$ (red) and combined $d f \mathbf{2}$ and $d f 3$ (green) at nine selected electrodes (left panel). Yellow bars indicate time windows with significant differences between the combined $d f 2$ and $d f 3$ and the $d f 1$ conditions as determined by the bootstrap BCa method (see the Method section). Right panel: Spline-interpolated voltage topographical maps for the combined $d f \mathbf{2}$ and $d f \mathbf{3}$ and the $d f \mathbf{1}$ conditions and difference maps [diff ( $d f \mathbf{2}$ and $d f \mathbf{3}$ minus $d f \mathbf{1})$ ] at representative time points of significant differences between conditions in question.

tratrial jitter would reduce the ERP response in the choice condition ( $d f 2$ and $d f 3$ ), as compared with the no-choice condition $(d f 1)$ where one predetermined task set had to be selected (cue phase). In contrast to ERPs, a jitter in the range of milliseconds would not affect the amplitude of the recorded BOLD signal, due to the poor temporal resolution of fMRI.

Our findings are also in line with electrophysiological studies investigating set switching (Rushworth, Passingham, \& Nobre, 2002) and attentional switching (Rushworth et al., 2005). These studies showed differential modulations over frontal and parietal sites when subjects were required to implement intentional or attentional sets, respectively. In several ERP experiments, Rushworth and colleagues (Rushworth, Hadland, Paus, \& Sipila, 2002; Rushworth, Passingham, \& Nobre, 2002) used indirect cue-task associations to indicate an upcoming switch or repeat trial. As with the procedure in the present study, these cues were not directly associated with a specific stimulus or response, so that no concrete action could be planned during the long CTI. Moreover, the length of the CTI was comparable to that used in the present study, leaving ample time for potential selection and reconfiguration processes to take place in advance of the presentation of the target. The studies by Rushworth and colleagues showed a comparable modulation with respect to cue-related processes, in that the set initiation phase elicited frontal activation (i.e., the source analyses indicated a dipole in the medial frontal wall) that was followed by parietal modulations subserving the implementation of concrete S-R mappings. Brass, Ullsperger, Knoesche, von Cramon, and Phillips (2005) showed that frontal activations can precede activations in parietal regions. This result is in line with hierarchical models stating that prefrontal areas might send a biasing signal to parietal regions to implement concrete S-R mappings (Miller \& Cohen, 2001; Tomita et al., 1999).

At first sight, the results of the present study revealed a different temporal involvement of frontal and parietal activation, in that the parieto-occipital activation was elicited about $200 \mathrm{msec}$ earlier (about $350 \mathrm{msec}$ after cue presentation) than the medial frontal modulation (about $550 \mathrm{msec}$ after cue presentation). We believe that the discrepancy with earlier studies is due to the fact that the earlier differential activation over parieto-occipital sites reflects a categorization process that is usually not manipulated in classical task-switching studies. Bledowski et al. (2006; Bledowski et al., 2004) argued that modulations over these sites reflect evaluation of the stimulus category. With respect to the present study, the presentation of the $2 \times 2$ grid at the beginning of a trial might induce a process that evaluates the number of options in choice (i.e., whether the trial is choice or no choice) and/ or the categorization of tasks. This categorization process is not needed in classical task-switching studies, because in these studies, subjects do not have the option to choose among different alternatives but, instead, are always unequivocally told what to do.

Ample evidence from neuroimaging, lesion, and electrophysiological studies suggests that medial frontal areas play a pivotal role in action selection (Ridderinkhof, Ullsperger, Crone, \& Nieuwenhuis, 2004; Rushworth, Behrens, Rudebeck, \& Walton, 2007; Rushworth, Walton, Kennerly, \& Bannerman, 2004) and, more specifically, in voluntary action selection (see, e.g., Lau, Rogers, Ramnani, \& Passingham, 2004; Walton, Devlin, \& Rushworth, 2004). In the latter studies, RCZ activation was found in conditions in which subjects could voluntarily select a movement or a response set, respectively. In the present study, the frontal modulation that follows the detection of the choice/no-choice option most likely reflects the selection of a specific task set, corroborating the results of the previous fMRI study (Forstmann et al., 2006).

Moreover, several authors have argued that the pMFC is involved in performance-monitoring processes such as the resolution of response conflict (Botvinick, Nystrom, Fissel, Carter, \& Cohen, 1999; Carter et al., 1998) or the 
detection of errors (Coles, Scheffers, \& Holroyd, 2001; Falkenstein, Hoormann, Christ, \& Hohnsbein, 2000; Ullsperger \& von Cramon, 2001). As in our previous fMRI study, we were interested in whether performancemonitoring processes might account for the present behavioral data pattern. Therefore, we computed congruency analyses for the S-R mappings for the $d f 2$ and $d f 3$ conditions. The results revealed no difference in RTs between task-set-incongruent and task-set-congruent choice trials. Hence, it appears unlikely that the differential MFC activation in choice conditions, as compared with the nochoice condition, was caused by task set congruency effects. Note, however, that it was not possible to compute the task set incongruency effects on ERP data, because the trial counts needed to keep the present design comparable to the previous fMRI experiment did not yield enough observations for a separate ERP analysis of congruent and incongruent trials. In sum, the present data appear to support the view that, in the context of volitional control, the pMFC is involved in voluntary task set selection, rather than in conflict detection.

Finally, in anticipation of the target, a sustained parietal negativity for the no-choice condition, as compared with the choice conditions, was observed that partly overlapped with the presentation of the target. The late negativity is comparable to the $\mathrm{CNV}$ wave, a slow negative brain potential that is typically recorded in the interval between two successive stimuli (Walter, Cooper, Aldridge, McCallum, \& Winter, 1964). The CNV has been observed in a variety of task-switching studies and is taken to reflect processing related to response preparation and stimulus anticipation in switch and repeat trials (Karayanidis, Coltheart, Michie, \& Murphy, 2003; van Boxtel \& Brunia, 1994). More specifically, several authors have proposed that the $\mathrm{CNV}$ might reflect a combination of maintenance and updating of S-R contingencies prior to the target presentation (see, e.g., Tieges et al., 2006; van Boxtel \& Brunia, 1994). Rushworth, Passingham, and Nobre (2002) and Wylie, Javitt, and Foxe (2003) reported a negative-going component that is similar to ours, albeit with a more frontal distribution.

Taken together, the cue-related and anticipatory-related activity resembles that of the activation sites in the previously conducted fMRI experiment. Moreover, several transient and sustained activations can be disentangled, shedding light on the underlying processes. Most important, the results indicate that parieto-occipital activation can precede frontal activation when there is a need to more precisely categorize the choice options at hand.

\section{Target-Related Activity}

The target evoked a series of ERP components that differed in amplitude between choice and no-choice conditions, including the N210-P210 - that is, a posterior lateral N210 and its polarity reversed fronto-central P210 (Joyce \& Rossion, 2005; Schendan \& Kutas, 2007) - and a centro-parietal P3 component. There is broad evidence that both components are involved in target-related processing (e.g., Kok, 2001). The N210-P210 complex has been argued to reflect early sensory-perceptual process- ing (Schendan \& Kutas, 2007). For example, Schendan and Kutas have provided evidence that the N210-P210 reflects an initial processing stage (i.e., a fast feedforward sweep that supports lower order image classification abilities) during which object parts are activated. With respect to the present findings, we argue that the presentation of the multivalent target might have induced the need to activate the relevant stimulus dimension in accordance with the chosen task during the cue period.

Following the N210-P210 complex, a P3 component was observed. In general, the $\mathrm{P} 3$ complex has been associated with the selection and categorization process of events (see, e.g., Barceló, Muñoz-Céspedes, Pozo, \& Rubia, 2000; Barceló, Periáñez, \& Knight, 2002; Kok, 2001)that is, the decision as to whether an event matches or does not match its internal representation. So, for example, the P3 amplitude was modulated by the number of task rules or sensory items held in memory (Barceló et al., 2002; Bledowski et al., 2006). Most notably, electrophysiological evidence from task-switching studies has also shown a smaller P3 amplitude for switch than for repeat trials (e.g., Kieffaber \& Hetrick, 2005; Tieges et al., 2006), perhaps reflecting the higher task difficulty of switch trials (see, e.g., Kok, 2001). Similar effects of switching in P3-like components have been reported by others (e.g., Karayanidis et al., 2003; Rushworth, Passingham, \& Nobre, 2002). In accordance with previous findings, we suggest that the present data pattern reflects a decision process of the relevant response that seems to be modulated by the number of options to choose from.

\section{Conclusion}

In the present study, we set out to investigate the temporal dynamics of voluntary selection processes, using ERPs. In a previous fMRI study, we observed that the RCZ and posterior parietal cortex were involved in voluntarily selecting among different task sets in choice conditions, as compared with the no-choice condition (Forstmann et al., 2006). The aim of the present study was to delineate the dynamics of proactive (cue-related) and reactive (targetrelated) processes involved in agency.

The results revealed modulations of an early transient parieto-occipital activation, taken to reflect a categorization process for the choice condition, as compared with the no-choice condition. This activation was followed by a transient fronto-central modulation subserving the voluntary selection between task sets, corroborating the findings of the previous fMRI study. Subsequently, in anticipation of the target, a CNV-like sustained negativity was found to be modulated by agency. This component is typically found in task-switching studies, reflecting preparatory processes in task reconfiguration. Notably, the present results indicated that the parieto-occipital activation preceded the frontal activation. This is taken as evidence for an additional categorization process being inserted when there is an option to choose or not to choose. This kind of categorization is usually not required in task-switching studies in which subjects are always unequivocally told what to do. Hence, the present data extend hierarchical models of the prefrontal cortex in that parieto-occipital activation can 
lead to frontal activation when more choice options are at hand.

The presentation of the target elicited a N210-P210 complex, followed by a P3. Both components were modulated by agency. The posterior N210 with its polarityreversed fronto-central P210 (Joyce \& Rossion, 2005) has been interpreted to reflect early perceptual processing. The P3 has been described extensively in electrophysiological studies investigating task switching and attentional switching. In the present study, we suggest that the N210P210 complex reflects an initial activation of the relevant stimulus dimension, which is followed by the P3 to reflect the categorical decision of the relevant $\mathrm{S}-\mathrm{R}$ rule.

In sum, the present study corroborates and extends the findings from a previously accomplished fMRI study (Forstmann et al., 2006). More specifically, the results revealed several subprocesses linked to cue and target presentation that could not be detected using fMRI.

\section{AUTHOR NOTE}

The work of B.U.F. and K.R.R. was supported by a VICI grant from the Netherlands Organization for Scientific Research (NWO) to K.R.R. We thank M. Geis-Dogruel and K. Spira for their support in data acquisition and M. Wibral and B. Rahm for advice on statistical data analyses. We also thank J. Neumann for helpful discussions on an earlier draft. Correspondence concerning this article should be addressed to B. U. Forstmann, Department of Psychology, University of Amsterdam, Roetersstraat 15, 1018 WB Amsterdam, The Netherlands (e-mail: b.u.forstmann@uva.nl).

\section{REFERENCES}

Barceló, F., Muñoz-Céspedes, J. M., Pozo, M. A., \& Rubia, F. J. (2000). Attentional set shifting modulates the target P3b response in the Wisconsin card sorting test. Neuropsychologica, 38, 1342-1355.

Barceló, F., Periáñez, J. A., \& Knight, R. T. (2002). Think differently: A brain orienting response to task novelty. NeuroReport, 13, 18871892.

Bledowski, C., Cohen Kadosh, K., Wibral, M., Rahm, B., BittNer, R. A., Hoechstetter, K., et AL. (2006). Mental chronometry of working memory retrieval: A combined functional magnetic resonance imaging and event-related potentials approach. Journal of Neuroscience, 26, 821-829.

Bledowski, C., Prvulovic, D., Hoechstetter, K., Scherg, M., Wibral, M., Goebel, R., \& Linden, D. E. (2004). Localizing P300 generators in visual target and distractor processing: A combined event-related potential and functional magnetic resonance imaging study. Journal of Neuroscience, 24, 9353-9360.

Botvinick, M., Nystrom, L. E., Fissel, K., Carter, C. S., \& Cohen, J. D. (1999). Conflict monitoring versus selection-for-action in anterior cingulate cortex. Nature, 402, 179-181.

Brass, M., Derrfuss, J., Forstmann, B., \& von Cramon, D. Y. (2005). The role of the inferior frontal junction area in cognitive control. Trends in Cognitive Sciences, 9, 314-316.

Brass, M., Ullsperger, M., Knoesche, T. R., von Cramon, D. Y., \& Phillips, N. A. (2005). Who comes first? The role of the prefrontal and parietal cortex in cognitive control. Journal of Cognitive Neuroscience, 17, 1367-1375.

Brass, M., \& VON CRAMON, D. Y. (2002). The role of the frontal cortex in task preparation. Cerebral Cortex, 12, 908-914.

Brass, M., \& von Cramon, D. Y. (2004). Decomposing components of task preparation with functional magnetic resonance imaging. Journal of Cognitive Neuroscience, 16, 609-620.

Braver, T. S., Gray, J. R., \& Burgess, G. C. (2007). Explaining the many varieties of working memory variation: Dual mechanisms of cognitive control. In A. R. A. Conway, C. Jarrold, M. J. Kane, A. Miyake, \& J. N. Towse (Eds.), Variation in working memory (pp. 76-106). Oxford: Oxford University Press.
Carter, C. S., Braver, T. S., Barch, D. M., Botvinick, M. M., Noll, D., \& CoHEN, J. D. (1998). Anterior cingulate cortex, error detection, and the online monitoring of performance. Science, 280, 747-749.

Coles, M. G. H., Scheffers, M. K., \& Holroyd, C. B. (2001). Why is there an ERN/Ne on correct trials? Response representations, stimulus-related components, and the theory of error-processing. Biological Psychology, 56, 173-189.

Corbetta, M., Kincade, J. M., Ollinger, J. M., McAvoy, M. P., \& Shulman, G. L. (2000). Voluntary orienting is dissociated from target detection in human posterior parietal cortex. Nature Neuroscience, $\mathbf{3}$, 292-297.

Corbetta, M., \& Shulman, G. L. (2002). Control of goal-directed and stimulus-driven attention in the brain. Nature Reviews Neuroscience, 3, 201-215.

Derrfuss, J., Brass, M., Neumann, J., \& von Cramon, D. Y. (2005). Involvement of the inferior frontal junction in cognitive control: Metaanalyses of switching and Stroop studies. Human Brain Mapping, 25, 22-34.

Duncan, J., \& OWEn, A. M. (2000). Common regions of the human frontal lobe recruited by diverse cognitive demands. Trends in Neurosciences, 23, 475-483.

Efron, B., \& Tibshirani, R. J. (1993). An introduction to the bootstrap. New York: Chapman \& Hall.

Falkenstein, M., Hoormann, J., Christ, S., \& Hohnsbein, J. (2000). ERP components on reaction errors and their functional significance: A tutorial. Biological Psychology, 51, 87-107.

Fink, G. R., Dolan, R. J., Halligan, P. W., Marshall, J. C., \& Frith, C. D. (1997). Space-based and object-based visual attention: Shared and specific neural domains. Brain, 120, 2013-2028.

Forstmann, B. U., Brass, M., Koch, I., \& von Cramon, D. Y. (2005). Internally generated and directly cued task sets: An investigation with fMRI. Neuropsychologia, 43, 943-952.

Forstmann, B. U., Brass, M., Koch, I., \& von Cramon, D. Y. (2006). Voluntary selection of task sets revealed by functional magnetic resonance imaging. Journal of Cognitive Neuroscience, 18, 388-398.

Foucher, J. R., Otzenberger, H., \& Gounot, D. (2003). The BOLD response and the gamma oscillations respond differently than evoked potentials: An interleaved EEG-fMRI study. BMC Neuroscience, 4, 22.

Huettel, S. A., McKeown, M. J., Song, A. W., Hart, S., Spencer, D. D., Allison, T., \& McCarthy, G. (2004). Linking hemodynamic and electrophysiological measures of brain activity: Evidence from functional MRI and intracranial field potentials. Cerebral Cortex, 14, $165-173$.

Ille, N., Berg, P., \& Scherg, M. (2002). Artifact correction of the ongoing EEG using spatial filters based on artifact and brain signal topographies. Journal of Clinical Neurophysiology, 19, 113-124.

Joyce, C., \& Rossion, B. (2005). The face-sensitive N170 and VPP components manifest the same brain processes: The effect of reference electrode site. Clinical Neurophysiology, 116, 2613-2631.

Karayanidis, F., Coltheart, M., Michie, P. T., \& Murphy, K. (2003). Electrophysiological correlates of anticipatory and poststimulus components of task switching. Psychophysiology, 40, 329-348.

Kastner, S., Pinsk, M. A., De Weerd, P., Desimone, R., \& UngerLEIDER, L. G. (1999). Increased activity in human visual cortex during directed attention in the absence of visual stimulation. Neuron, 22, 751-761.

Kieffaber, P. D., \& Hetrick, W. P. (2005). Event-related potential correlates of task switching and switch costs. Psychophysiology, 42, 56-71.

KocH, I. (2003). The role of external cues for endogenous advance reconfiguration in task switching. Psychonomic Bulletin \& Review, 10, 488-492.

KoK, A. (2001). On the utility of P3 amplitude as a measure of processing capacity. Psychophysiology, 38, 557-577.

LaU, H. C., Rogers, R. D., Ramnani, N., \& Passingham, R. E. (2004). Willed action and attention to the selection of action. NeuroImage, 21, 1407-1415.

MeIran, N. (1996). Reconfiguration of processing mode prior to task performance. Journal of Experimental Psychology: Learning, Memory, \& Cognition, 22, 1423-1442.

Miller, E. K., \& CoHEN, J. D. (2001). An integrative theory of prefrontal cortex function. Annual Review of Neuroscience, 24, 167-202.

Nieuwenhuis, S., Aston-Jones, G., \& Cohen, J. D. (2005). Decision 
making, the $\mathrm{P} 3$, and the locus coeruleus-norepinephrine system. Psychological Bulletin, 131, 510-532.

Nobre, A. C., Sebestyen, G. N., Gitelman, D. R., Mesulam, M. M., Frackowiak, R. S., \& Frith, C. D. (1997). Functional localization of the system for visuospatial attention using positron emission tomography. Brain, 120, 515-533.

PicARD, N., \& StRick, P. L. (1996). Motor areas of the medial wall: A review of their location and functional activation. Cerebral Cortex, 6, 342-353.

Ridderinkhof, K. R., Ullsperger, M., Crone, E. A., \& NieuwenHUIS, S. (2004). The role of the medial frontal cortex in cognitive control. Science, 306, 443-447.

Ridderinkhof, K. R., van den Wildenberg, W. P., Segalowitz, S. J., \& CARTER, C. S. (2004). Neurocognitive mechanisms of cognitive control: The role of prefrontal cortex in action selection, response inhibition, performance monitoring, and reward-based learning. Brain \& Cognition, 56, 129-140.

Rogers, R. D., \& Monsell, S. (1995). Costs of a predictable switch between simple cognitive tasks. Journal of Experimental Psychology: General, 124, 207-231.

Ruge, H., Brass, M., Koch, I., Rubin, O., Meiran, N., \& von CraMON, D. Y. (2005). Advance preparation and stimulus-induced interference in cued task switching: Further insights from BOLD fMRI. Neuropsychologia, 43, 340-355.

Rushworth, M. F. S., Behrens, T. E. J., Rudebeck, P. H., \& Walton, M. E. (2007). Contrasting roles for cingulate and orbitofrontal cortex in decisions and social behaviour. Trends in Cognitive Sciences, 11, 168-176.

Rushworth, M. F. S., Hadland, K. A., Paus, T., \& Sipila, P. K. (2002). Role of the human medial frontal cortex in task switching: A combined fMRI and TMS study. Journal of Neurophysiology, 87, 2577-2592.

Rushworth, M. F. S., Passingham, R. E., \& Nobre, A. C. (2002). Components of switching intentional set. Journal of Cognitive Neuroscience, 14, 1139-1150.

Rushworth, M. F. S., Passingham, R. E., \& Nobre, A. C. (2005). Components of attentional set-switching. Experimental Psychology, 52, 83-98.
Rushworth, M. F. S., Walton, M. E., Kennerly, S. W., \& BannerMAN, D. M. (2004). Action sets and decisions in the medial frontal cortex. Trends in Cognitive Sciences, 8, 410-417.

Schendan, H. E., \& Kutas, M. (2007). Neurophysiological evidence for the time course of activation of global shape, part, and local contour representations during visual object categorization and memory. Journal of Cognitive Neuroscience, 19, 734-749.

Tieges, Z., Snel, J., KoK, A., Wijnen, J. G., Lorist, M. M., \& RidDERINKHOF, K. R. (2006). Caffeine improves anticipatory processes in task switching. Biological Psychology, 73, 101-113.

Tomita, H., Ohbayashi, M., Nakahara, K., Hasegawa, I., \& MiyaSHITA, Y. (1999). Top-down signal from prefrontal cortex in executive control of memory retrieval. Nature, 401, 699-703.

Ullsperger, M., \& von Cramon, D. Y. (2001). Subprocesses of performance monitoring: A dissociation of error processing and response competition revealed by event-related fMRI and ERPs. NeuroImage, 14, 1387-1401.

van Boxtel, G. J. M., \& Brunia, C. H. M. (1994). Motor and nonmotor components of the contingent negative variation. International Journal of Psychophysiology, 17, 269-279.

VERLEGER, R. (1997). On the utility of P3 latency as an index of mental chronometry. Psychophysiology, 34, 131-156.

Walter, W., Cooper, R., Aldridge, V., McCallum, W., \& Winter, A. (1964). Contingent negative variation: An electric sign of sensorimotor association and expectancy in the human brain. Nature, 203, 380-384.

Walton, M. E., Devlin, J. T., \& Rushworth, M. F. S. (2004). Interactions between decision making and performance monitoring within prefrontal cortex. Nature Neuroscience, 7, 1259-1265.

Wylie, G. R., JavitT, D. C., \& Foxe, J. J. (2003). Task switching: A high-density electrical mapping study. NeuroImage, 20, 2322-2342.

WyLie, G. R., Javitt, D. C., \& FoXe, J. J. (2004). Don't think of a white bear: An fMRI investigation of the effects of sequential instructional sets on cortical activity in a task-switching paradigm. Human Brain Mapping, 21, 279-297.

(Manuscript received February 3, 2007; revision accepted for publication September 14, 2007.) 